\title{
Estrutura populacional de Portunus spinimanus Latreille (Crustacea, Portunidae) na Armação do Itapocoroy, Penha, Santa Catarina, Brasil
}

\author{
Joaquim Olinto Branco ${ }^{1}$ \\ Maria José Lunardon-Branco ${ }^{1}$ \\ Flávio Xavier Souto ${ }^{1}$
}

\begin{abstract}
Population structure of Portunus spinimanus Latreille (Crustacea, Portunidae) in the Armação do Itapocoroy, Penha, Santa Catarina, Brazil. Portunus spinimanus Latreille, 1819 is one abundant Portunidae species which are caught by sea-bob-shrimp's artisanal fishery in Armação do Itapocoroy, Penha, Santa Catarina, Brazil. Aiming to analyze its population structure, a total of 995 males and 1925 females were collected by means of overtrawl from January to December 1995 , between the depths of 6 to 10 meters, in the periods of morning, afternoon and evening. The width variation of the carapace ranged from 3.0 to $14.0 \mathrm{~cm}$ in the males and 3.0 to $11.0 \mathrm{~cm}$ in females. The species presents growth isometric and the males reach width and weight assintotic and bigger that the females. The estimated first maturation width was between 6.8 and $7.6 \mathrm{~cm}$, reached with the approximated age of 15 months. The artisanal fishery in Armação do Itapocoroy is acting with a higher intensity over the juveniles stock.

KEY WORDS. Portunus spinimanus, population structure, artisanal fishery
\end{abstract}

O siri-candeia ou siri-canela Portunus spinimanus Latreille, 1819, apresenta ampla distribuição geográfica no Atlântico Ocidental, ocorrendo de New Jersey (EUA) ao Estado do Rio Grande do Sul (Brasil), onde habita águas salobras de canais e baías com fundos de areia, lama e conchas quebradas; são capturados a partir da zona de maré até $90 \mathrm{~m}$ de profundidade (MELO 1996).

Apesar da sua freqüência nos arrastos dirigidos à pesca de camarão, tamanho e sabor da carne adequados ao consumo humano, existem poucas informações sobre a biologia da espécie no litoral brasileiro (MESQUITA 1972; SANTOS et al. 1995a). Este trabalho tem como objetivo caracterizar a estrutura populacional de $P$. spinimanus na Armação do Itapocoroy, Penhas (Santa Catarina), fornecendo subsídios para uma exploração racional desse recurso.

\section{MATERIAL E MÉTODOS}

Durante o período de janeiro a dezembro de 1995 foram realizadas coletas mensais nos turnos da manhã, tarde e noite, e a cada estação do ano, coletas de 24 horas com intervalo de 4 horas entre os arrastos na Armação do Itapocoroy, Penha $\left(26^{\circ} 46^{\prime}-26^{\circ} 47^{\prime} \mathrm{S}\right.$ e $\left.48^{\circ} 36^{\prime}-48^{\circ} 37^{\prime} \mathrm{W}\right)$. A profundidade na área dos arrastos variou entre 6,0 a $10,0 \mathrm{~m}$, com fundo arenoso nas partes rasas e com sedimento

1) Centro de Ciências Tecnológicas da Terra e do Mar, UNIVALI. Caixa Postal 360, 88301-970 Itajaí, Santa Catarina, Brasil. E-mail: branco@cttmar.univali.br 
biodetrítico nas mais profundas. Foram utilizadas duas redes-de-arrasto com portas, com 6,0 $\mathrm{m}$ de abertura de boca, malha de 3,0 $\mathrm{cm}$ entre nós na manga e no corpo e $2,0 \mathrm{~cm}$ no ensacador. O tempo de cada arrasto foi de 30 minutos com velocidade média da baleeira de dois nós.

Foram registrados dados de temperatura $\left({ }^{\circ} \mathrm{C}\right)$ e salinidade $(\%$ ) da água de fundo e de cada exemplar, o sexo, estágio de maturação (juvenil e adulto) pelo formato e aderência do abdome aos esternitos torácicos (TAISSOUN 1969), largura total da carapaça (Wid) em cm e o peso total (Wt) em gramas.

Para verificar a possível diferença entre os sexos, mensalmente e por classe de comprimento, utilizou-se o teste do $X^{2}$ com nível de significância de 0,05 e (n-1) graus de liberdade. A relação peso/largura total foi calculada para cada sexo separadamente, de acordo com SANTOS (1978). O tamanho de primeira maturação (WidPM), foi estimado segundo VAZZOLER (1981). Para verificar a atuação da pesca artesanal do camarão sobre a população de $P$. spinimanus, utilizou-se a distribuição de freqüência por classe de comprimento para machos e fêmeas, baseando-se na estimativa do tamanho de primeira maturação gonadal.

As curvas de crescimento em largura total da carapaça foram estimadas para machos e fêmeas pelo método da distribuição de frequência. A validade da expressão de BERTALANFFY (1938) foi testada previamente, com a aplicação da transformação de WALFORD (1946) e os parâmetros estimados conforme SANTOS (1978). A ANOVA $(p<0,05)$ foi utilizada para verificar a influência do período de coleta ou da estação do ano na abundância de $P$. spinimanus.

\section{RESULTADOS}

A temperatura da água de fundo nos três períodos de coleta, apresentou uma flutuação sazonal com valores mais elevados no verão $\left(25,3 \pm 1,5^{\circ} \mathrm{C}\right)$ e na primavera $\left(25,2 \pm 1,8^{\circ} \mathrm{C}\right)$, e mais baixos no outono $\left(21,0 \pm 1,6^{\circ} \mathrm{C}\right)$ e inverno $\left(20,2 \pm 1,2^{\circ} \mathrm{C}\right)$ (Fig. 1a). O padrão de variação da temperatura manteve-se uniforme entre os turnos de amostragens.

A salinidade apresentou pequenas oscilações ao longo do ano entre os períodos de coleta (Fig. 1b), com valores médios mais elevados na primavera $(33,7 \pm 1,1 \%$ o e inverno $(33,1 \pm 1,6 \%$ e e menores no verão $(33,7 \pm 1,1 \%$ ) e inverno $(31,4 \pm 1,8 \%$ ) (Fig. 1b).

Para o total de $P$. spinimanus capturados, as frequiências relativas das fêmeas $(65,5 \%)$ e dos machos $(34,5 \%)$ foram diferentes do esperado de 1:1 na população (Fig. 2a). Essa tendência ficou evidente com a aplicação do teste do $X^{2}$ por mês de coleta, indicando diferenças significativas a favor das fêmeas a partir de maio até dezembro (Fig. 2a). No restante do período amostral, foi observado um equilíbrio na proporção sexual da população.

As distribuições das frequiências de machos e fêmeas por classe de largura total da carapaça, apresentaram uma amplitude de 3,0 a 14,0 $\mathrm{cm}$ nos machos e de 3,0 a $11,0 \mathrm{~cm}$ para as fêmeas (Fig. 2b); com diferença significativa em favor das fêmeas entre as classes de 6,0 a $8,0 \mathrm{~cm}$, e dos machos entre 10,0 a 11,0 cm. 

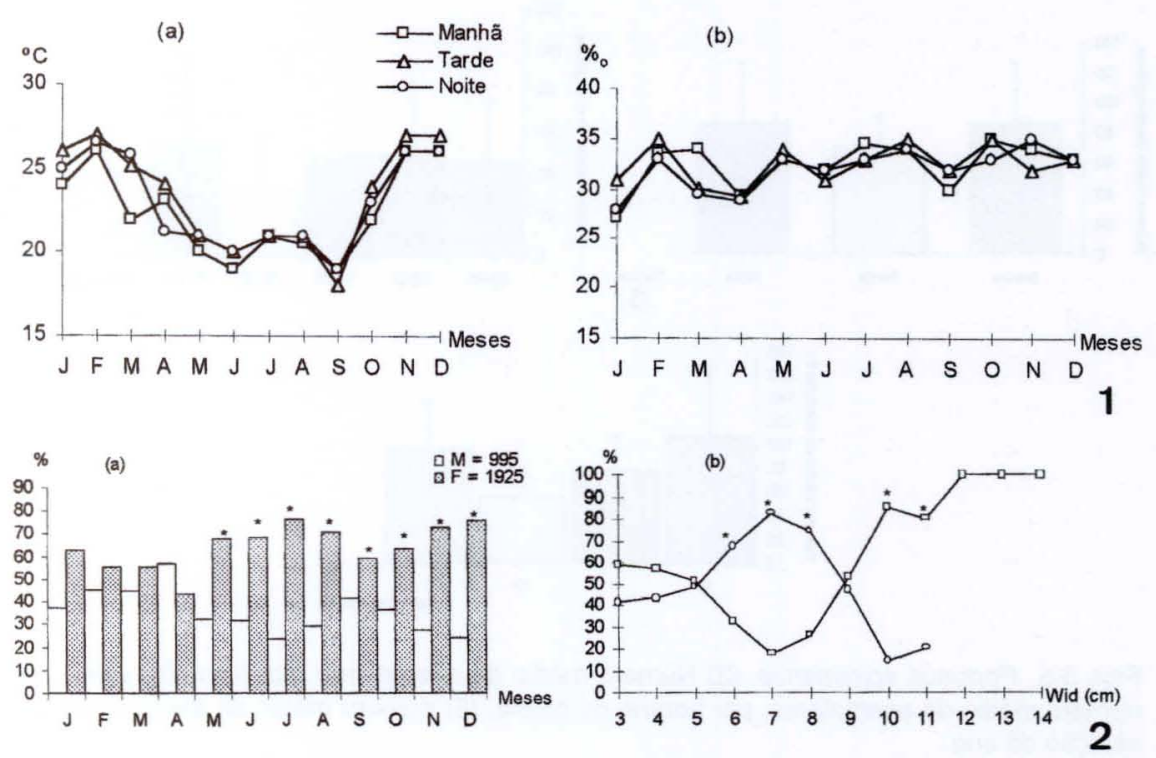

Figs 1-2. (1) Variações mensais da temperatura (a) e salinidade (b) da água de superfície; (2) Freqüência de ocorrência (\%) de Portunus spinimanus por mês (a) e classe de largura da carapaça (b). (círculo) Fêmeas, (quadrado) machos.

Durante o período de estudo foram coletados 2920 exemplares sendo que a maior média mensal ocorreu durante o turno da noite $(86,4)$ seguido da manhã $(85,6)$, e da tarde $(71,3)$ (Fig. 3), sendo que as maiores capturas ocorreram no horário das 12:00, 16:00 e 24:00 horas (Fig. 4). Independente do turno e horário de coleta, a maior abundância foi registrada durante as estações de primavera e inverno (Fig. 5).

A ANOVA aplicada à abundância de Portunus spinimanus, por turno de coleta $(\mathrm{F}=0,509 ; \mathrm{gl} 2-33)$, horário $(\mathrm{F}=0,468 ; \mathrm{gl} 5-42)$ e estação do ano $(\mathrm{F}=$ 2,572; gl 3-32), não indicou diferença significativa.

Os valores do peso total foram lançados em gráfico em função da largura total da carapaça, sendo corroborados pela aderência dos pontos empíricos à curva e pelos valores do coeficiente de determinação ( $\mathrm{r}^{2}$ ) (Fig. 6a, b). A amplitude de variação de largura da carapaça nos machos foi de 3,0 a $14,0 \mathrm{~cm}$ e a do peso de 1,3 a $400 \mathrm{~g}$, enquanto nas fêmeas, a largura variou entre 3,0 a $11,0 \mathrm{~cm}$ e o peso entre 1,1 a $180,0 \mathrm{~g}$.

As curvas de crescimento em largura total foram descritas pelas equações: machos: Wid = 14,6 $\left(1-\mathrm{e}^{-0,6076 \mathrm{t}}\right)$; fêmeas: $\mathrm{Wid}=12,2\left(1-\mathrm{e}^{-0,5786 \mathrm{t}}\right)$. Na figura 7, encontram-se as curvas teóricas ajustadas aos pontos empíricos para machos e fêmeas. Portunus spinimanus apresentou uma constante catabólica elevada, sendo o maior valor da largura total da carapaça observado na população da Armação do Itapocoroy atingido em torno dos 22,0 meses de idade. 

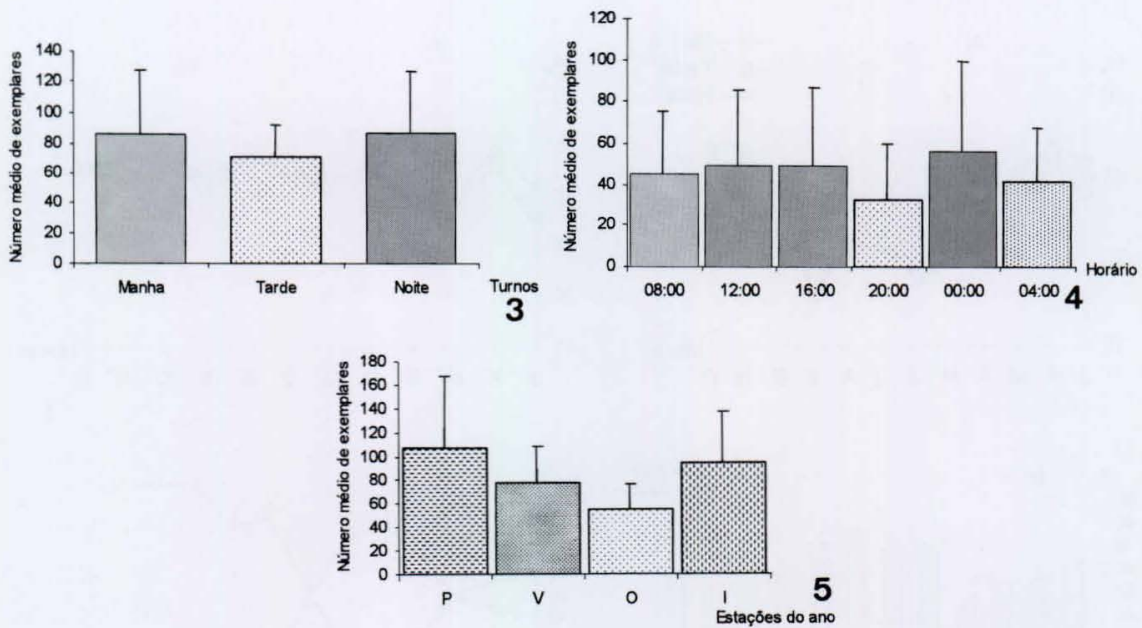

Figs 3-5. Portunus spinimanus. (3) Número médio de exemplares, por turno de coleta; (4) número médio de exemplares, por horário de coleta; (5) número médio de exemplares, por estação do ano.
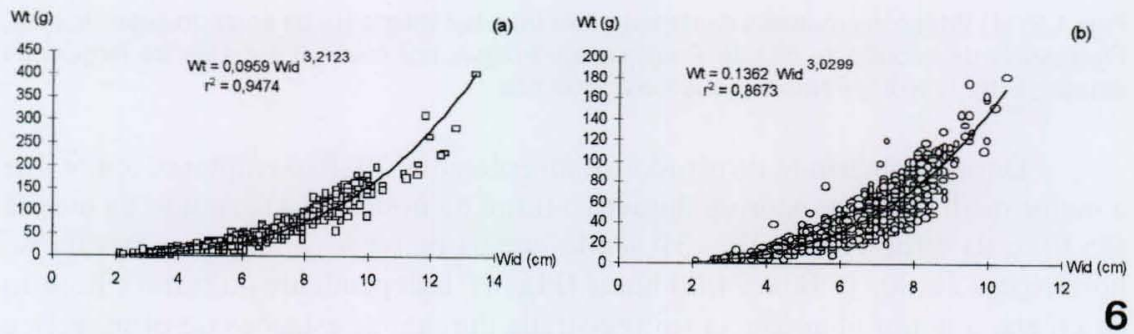

Wid $(\mathrm{cm})$

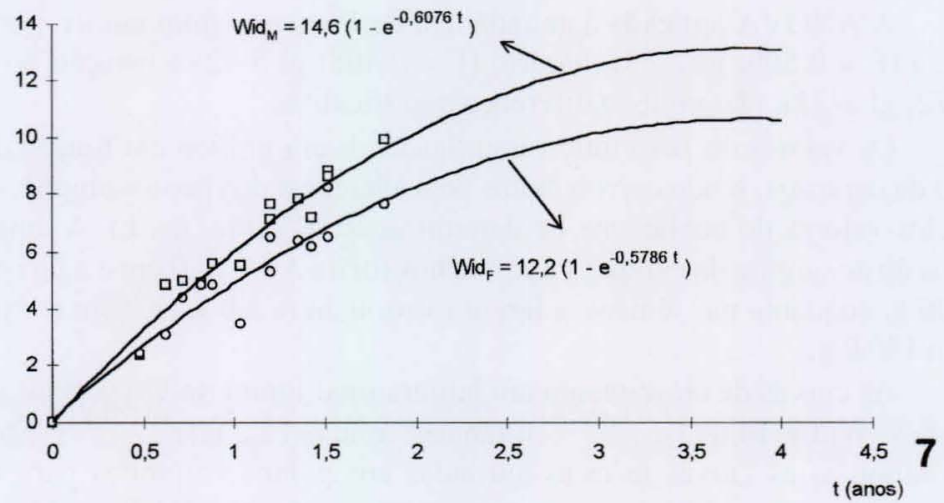

Figs 6-7. Portunus spinimanus. (6) Relação peso/largura da carapaça para macho (a) e fêmeas (b); (7) curva de crescimento em largura da carapaça. 
O tamanho de primeira maturação foi estimado em $6,8 \mathrm{~cm}$ de largura total de carapaça para as fêmeas e 7,6 cm para os machos; a partir de $11,0 \mathrm{~cm}$, todos os siris coletados eram adultos (Fig. 8). Analisando-se as distribuições anuais da frequiência de largura por turnos de coleta (Fig. 9), em função do tamanho de primeira maturação, verificou-se que a pesca artesanal na Armação do Itapocoroy atuou com maior intensidade sobre o estoque de juvenis ( machos $=64,7 \%$ e fêmeas $=62,6 \%$ ) e com menor intensidade sobre os adultos ( $\operatorname{machos}=35,3 \%$ e fêmeas $=37,4 \%$ ).

A análise da figura 10, indica que ocorreram flutuações sazonais na abundância das fêmeas de $P$. spinimanus; sendo que nas estações de primavera e verão foram registradas as maiores capturas de fêmeas imaturas, enquanto que nas estações de inverno e primavera ocorreram as maiores freqüência das fêmeas maturas e ovígeras (Fig. 10).

\section{DISCUSSÃO}

O padrão sazonal da temperatura da água de superfície na Armação do Itapocoroy, está de acordo com o esperado para a região (MATSUURA 1986); enquanto que as flutuações nos teores de salinidade podem ser associados à contribuição fluvial do Rio Itajaí-Açú, cuja foz está localizada aproximadamente a $20 \mathrm{~km}$ ao sul da área de estudo (BRANCO et al. 1999). Para SANTOS et al. (1994) a abundância de Portunus spinimanus na enseada da Fortaleza, Ubatuba (São Paulo), esteve significativamente associada com a profundidade, temperatura, salinidade e granulometria do sedimento. Embora a temperatura e a salinidade não tenham constituído um fator diferencial entre os períodos de amostragens, as maiores abundâncias relativas da espécie na área de estudo ocorreram nas estações de primavera e inverno.

A literatura disponível no país, não aborda a proporção sexual do siri-candeia, na Armação do Itapocoroy; a proporção esperada de 1:1 não foi mantida quando considerado o total da população. Mensalmente, confirmou-se essa disparidade com domínio significativo das fêmeas entre o período de maio a dezembro. Em relação ao tamanho, constatou-se o predomínio significativo das fêmeas entre os exemplares de 6,0 a $8,0 \mathrm{~cm}$ e dos machos entre 10,0 a $11,0 \mathrm{~cm}$.

A relação peso/comprimento tem sido utilizada para estimar o peso de um exemplar através do conhecimento da sua largura, sendo amplamente empregada em estudos de dinâmica populacional e avaliação de estoques. Portunus spinimanus é o representante dos Portunidae estudados na costa brasileira, até o momento, que apresentou os maiores valores de alometria positiva, variando entre 3,33 (machos) e 3,13 (fêmeas) no litoral de Ubatuba (SANTOS et al. 1995a) e 3,27 para sexos agrupados na costa cearense (MESQUITA 1972). Essa tendência, também foi observada na população de siri-candeia da Armação do Itapocoroy. Em geral, nos siris Portunidae, os machos apresentam largura da carapaça superior às das fêmeas, com taxas de crescimento próximas (BRANCO \& MASUNARI 1992), o que foi observado, também para a presente espécie.

O conhecimento do tamanho de primeira maturação gonadal é fundamental para o gerenciamento dos estoques em explotação, pois fornece a informação básica para a determinação do tamanho mínimo de captura e dimensionamento das malhas 

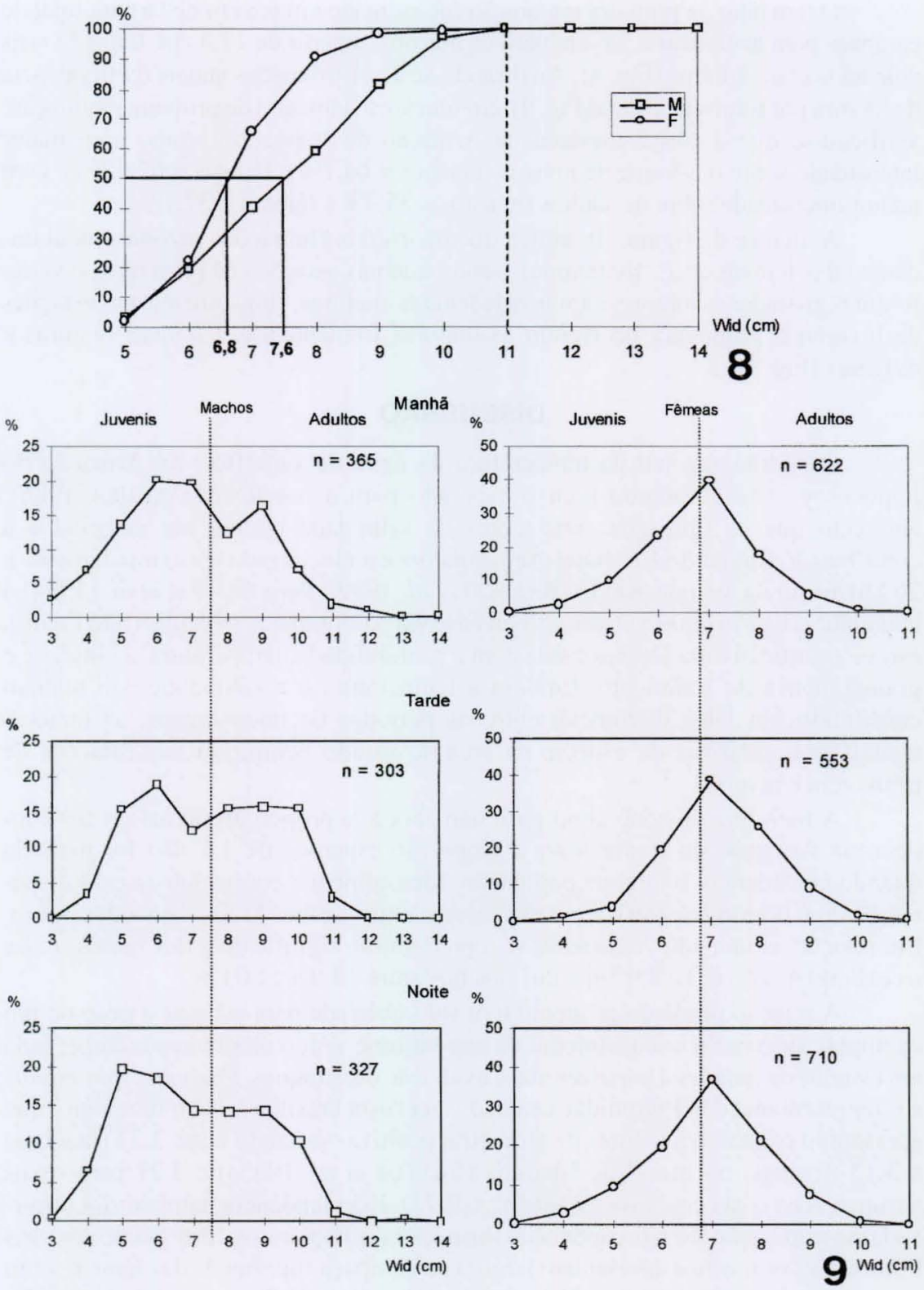

Figs 8-9. Distribuição de freqüência de Portunus spinimanus. (8) Machos e fêmeas adultas, por classe de largura da carapaça, machos: WidpM $=7,6 \mathrm{~cm}$, fêmeas: WidpM $=6,8 \mathrm{~cm}$; $(9)$ classes de largura da carapaça, por turno de coleta.

Revta bras. Zool. 19 (3): 731 - 738, 2002 


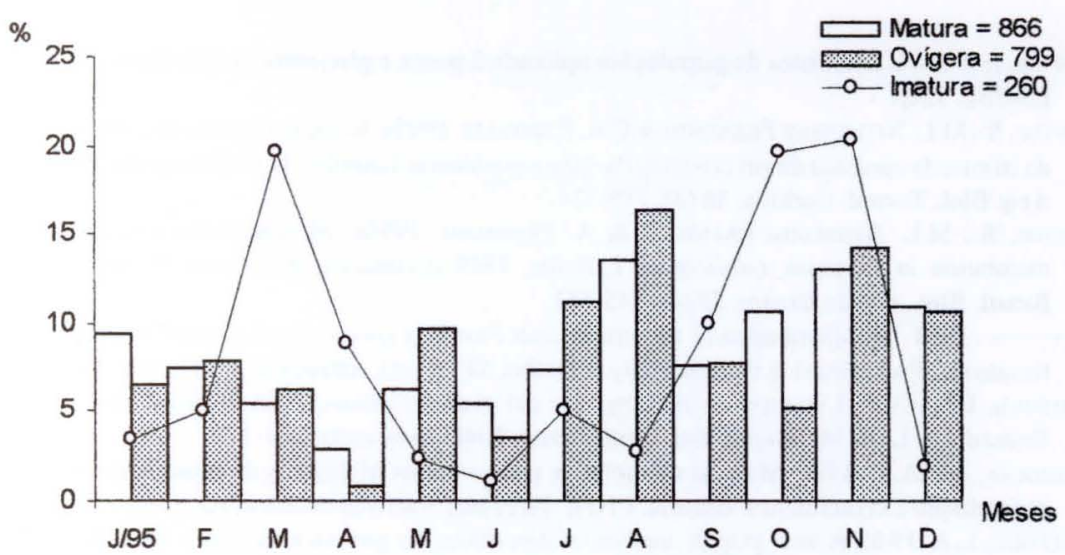

Fig. 10. Portunus spinimanus. Distribuição de freqüência de ocorrência (\%) das fêmeas maturas, ovígeras e imaturas.

das redes. Além disso, a sobreposição desse tamanho com as curvas de distribuição de comprimento ou largura da carapaça permitem determinar o estrato da população em que a pesca vem atuando com maior intensidade (BRANCO et al. 1999). A maior vulnerabilidade dos siri-candeia juvenis na pesca do camarão sete-barbas (Xiphopenaeus kroyeri Heller, 1862), provavelmente esteja relacionado com o tamanho de malha das redes de pesca.

Nesse estudo, as fêmeas de $P$. spinimanus atingiram tamanho de primeira maturação com $6,8 \mathrm{~cm}$ largura de carapaça e os machos com 7,6 cm. Esses tamanhos foram alcançados com idade em torno de 1,3 ano.

Embora SANTOS et al. (1995b), não apresentem os valores do tamanho de primeira maturação de $P$. spinimanus para o litoral de Ubatuba, esses podem ser deduzidos da relação comprimento/largura da carapaça, ficando em torno de 5,7 $\mathrm{cm}$. Provavelmente, essa diferença entre as populações de Ubatuba e Armação do Itapocoroy são inerentes aos processos de determinação ou padrões regionais de crescimento da espécie.

\section{REFERÊNCIAS BIBLIOGRÁFICAS}

BertalanfFy, L. VON. 1938. A quantitative theory of organic growth. Hum. Biol. 10 (2): 181-213.

Branco, J.O.; M.J. Lunardon-Branco; F.X. Souto \& C.R. Guerra. 1999. Estrutura populacional do camarão sete-barbas Xiphopenaeus kroyeri (Heller, 1862), na foz do Rio Itajaí-Açú, SC, Brasil. Braz. arch. biol. technol., Curitiba, 42(1): 115-126.

Branco, J.O. \& S. Masunari. 1992. Crescimento de Callinectes danae Smith (Decapoda, Portunidae) da Lagoa da Conceição, Florianópolis, Santa Catarina, Brasil. Revta bras. Zool., Curitiba, 9 (1/2): 53-66.

MAtsuUra, Y. (1986), Contribuição ao estudo da estrutura oçeanográfica da Região Sudeste entre Cabo Frio (RJ) e Cabo de Santa Marta Grande (SC).Ci. Cult., Rio de Janeiro, 38 (8): 1439-1450.

MELo, G.A.S. 1996. Manual de identificação dos Brachyura (caranguejos e siris) do litoral brasileiro. São Paulo: Plêide, FAPESP, 604p.

Mesquita, A.L.L. 1972. Dados biométricos do siri canela Portunus spinimanus Latreille, 1819 (Decapoda, Brachyura, Portunidae). Arq. Ci. Mar., Fortaleza, 12 (1): 88-90. 
SANTOS, E.P. 1978. Dinâmica de populações aplicada à pesca e piscicultura. São Paulo, HUCITEC/ EDUSP, 129p.

Santos, S.; M.L. Negreiros-Fransozo \& C.R. Padovani. 1995a. Relação do peso do corpo em função da largura da carapaça do siri candeias Portunus spinimanus Latreille, 1819 (Decapoda, Portunidae). Arq. Biol. Tecnol. Curitiba, 38 (3): 715-724.

Santos, S.; M.L. Negreiros-Fransozo \& A. Fransozo. 1995b. Morphometric relationships and maturation in Portunus spinimanusI Latreille, 1819 (Crustacea, Brachyura, Portunidae). Rev. Brasil. Biol., Rio de Janeiro, 55 (4): 545-553.

1994. The distribution of swimming crab Portunus spinimanus Latreille, 1819 (Crustacea, Brachyura, Portunidae) in Fortaleza Bay, Ubatuba, SP, Brazil. Atlântica, Rio Grande, 16: 125-141.

TAissoun, E.N. 1969. Los especies de cangrejos del genero Callinectes (Brachyura) en el Golfo de Venezuela e Lago Maracaibo. Bol. Cent. Invest. Biol., Venezuela, 2: 1-112.

VAzzoLER, A.E.A.M. 1981. Manual de métodos para estudos biológicos de populações de peixes: reprodução e crescimento. Brasília, CNPq, Programa Nacional de Zoologia, 106p.

WALFORD, L.A. 1946. A new graphic method of describing the growth of animals. Biol. Bull. 90 (2): 141-147.

Recebido em 20.XII.2001; aceito em 23.VII.2002. 